\title{
Clinical and epidemiological profile of neuroendocrine tumors: An experience from a regional cancer center from Western India
}

\author{
Rahul Suhas Kulkarni, Asha S.Anand, Sonia K. Parikh, Harsha P. Panchal, Apurva A. Patel, Dhruv P. Mehta, Priyanka Patel
}

\begin{abstract}
Background: Most of the data on neuroendocrine tumors (NETs) are from theWestern literature. Indian studies regarding clinicopathological characteristics and treatment outcomes are lacking. Methods: This is a prospective observational study of all new patients with NETs (except small-cell lung cancer) registered at our tertiary care cancer institute from November 2014 to November 2016. A total of 97 new patients were registered, of which 20 were lost to follow-up before starting any planned treatment. Epidemiological and clinicopathological features of all these 97 patients were studied, and the remaining 77 patients were analyzed for treatment response and survival analysis. Results: The median age at diagnosis was 49 years (20-74 years) with male preponderance $(\mathrm{M}: \mathrm{F}=\mathrm{I} .85: \mathrm{I})$. The most common primary site of origin was pancreas $(34 / 97=35 \%)$, followed by unknown primary origin ( $19 \%)$, small intestine (9\%), and pulmonary (6\%). Of 97 patients, 91 (93.8\%) presented with nonfunctional symptoms, 3 (3.1\%) had purely functional symptoms, and 3 (3.1\%) presented with both functional and nonfunctional symptoms. The most common presenting symptom was abdominal pain (59.7\%), followed by jaundice $(9.3 \%)$, whereas watery diarrhea $(83.3 \%)$ and flushing $(66.7 \%)$ were the most common functional symptoms. Sixty-six percent (64/97) of cases were metastatic at presentation. A strong correlation was noted between the primary site of origin and metastatic presentation $(P=0.016)$. Chemotherapy was the most common primary therapy $(40.2 \%)$, followed by surgery $(28.6 \%)$, watchful waiting ( $15.6 \%)$, and somatostatin analogs (II.7\%). The median event-free survival was highest for patients undergoing surgery (I0 months). Conclusions: The clinicopathological profile of NETs in the Indian population differs from Western countries. Majority of patients present with metastatic disease, thus representing a need for creating awareness among patients and medical fraternity and formulating Indian guidelines for optimized treatment.
\end{abstract}

Key words: Clinical profile, epidemiology, Indian data, neuroendocrine tumors, pathological features, treatment outcomes

\section{Introduction}

Neuroendocrine tumors (NETs) encompass a heterogeneous group of tumors arising from amine-producing neuroendocrine cells anywhere in the body. ${ }^{[1]}$ The incidence of NETs is 2 per lakh, and they account for $0.5 \%$ of all malignancies. ${ }^{[2]}$ Based on the ability to produce certain peptides, NETs are classified as being functional and nonfunctional. Histopathological classification of NETs has proven to be extremely difficult. In 2005 and 2006, the European Neuroendocrine Tumor Society proposed tumor-node-metastasis grading systems, in which proliferative indices were incorporated and NETs were classified from Grade 1 to Grade $3{ }^{[3,4]}$ In 2010 , the WHO validated this crucial role of proliferative rate, thus classifying gastroenteropancreatic (GEP) NETs into two categories: well differentiated (Grade 1 and 2) and poorly differentiated (Grade 3 ). ${ }^{[5]}$ Due to the enigmatic nature of NETs, various guidelines have been developed based on the national and institutional consensus committees. There is a serious lack of Indian studies regarding epidemiology, presentations, histopathological characteristics, and treatment outcomes in patients with NETs.

\section{Methods}

This is a prospective, observational study of 97 patients with histologically and immunohistochemistry (IHC)-proven diagnosis of NET. All new patients of NET diagnosed between November 1, 2014, and November 1, 2016, were included in the study $(n=97)$. Biopsy was performed either from the primary or from the metastatic lesion, and the biopsy specimen was analyzed with regard to the following points: tumor location and type, neuroendocrine morphology, grade of NET (MIB1 index), degree of differentiation, IHC for confirmation including synaptophysin and chromogranin A, and other markers as deemed appropriate for exclusion of other diagnoses. Patients were classified according to the WHO

\begin{tabular}{|l|}
\hline Access this article online \\
\hline Quick Response Code: \\
口) \\
Website: www.sajc.org \\
\hline DOI: 10.4103 /sajc.sajc_364_18 \\
\hline
\end{tabular}

Department of Medical and Pediatric Oncology, Gujarat Cancer Research Institute, Ahmedabad, Gujarat, India

Correspondence to: Dr.Asha S.Anand,

E-mail: ashaanand1757@yahoo.com
2010 classification. Patients were evaluated clinically and radiologically at regular intervals (after every 2-4 courses of planned treatment) or after every 3 months of surgery/watchful waiting and whenever deemed necessary. Patients were assessed for subjective response, objective response, and treatment-related toxicity. The response was assessed according to the RECIST 1.1 guidelines. All patients were included in the analysis of demography and clinical profile. However, only those patients who received minimum three courses of planned therapy (chemotherapy/octreotide long-acting release $[\mathrm{LAR}]$ ) and had a follow-up imaging evaluation after minimum 3 months $/ 3$ courses of planned treatment were included for treatment response analysis and survival analysis. Patients whose response evaluation was done after minimum 3 months $/ 3$ courses of planned treatment and later as per the protocol but who subsequently were lost to follow-up were included in the analysis of event-free survival (EFS). Thus, EFS was defined as the period from the start of planned therapy till the day of progression/death/lost to follow-up after planned response evaluation (the event was truncated at the last follow-up). All statistical tests were two-sided, with $P<0.05$ being considered statistically significant. Statistical analysis was performed using Epi Info software version 7 (available free online).

\section{Results}

\section{Demographics and tumor characteristics}

Of a total of 97 patients analyzed, the maximum number of patients belonged to the age group of 51-60 years (36.1\%). The median age at presentation was 49 years (range: 20-74 years), with a male-to-female ratio being 1.85:1. Pancreas was the most common primary site of origin (35\%), followed by unknown primary origin (19\%) and small intestine (9\%).

This is an open access journal, and articles are distributed under the terms of the Creative Commons Attribution-NonCommercial-ShareAlike 4.0 License, which allows others to remix, tweak, and build upon the work non-commercially, as long as appropriate credit is given and the new creations are licensed under the identical terms.

For reprints contact: reprints@ @medknow.com

How to cite this article: Kulkarni RS, Anand AS, Parikh SK, Panchal HP, Patel AA, Mehta DP, et al. Clinical and epidemiological profile of neuroendocrine tumors: An experience from a regional cancer center from Western India. South Asian J Cancer 2019;8: 198-202. 
Detailed primary site distribution is shown in Figure 1. No correlation was found between the primary site with age $(P=0.5)$ or tumor grade $(P=0.212)$.

Of 97 patients, 91 (93.8\%) presented with only nonfunctional symptoms, 3 (3.1\%) had purely functional symptoms, and $3(3.1 \%)$ presented with both functional and nonfunctional symptoms. Thus, only $6(6.2 \%)$ of 97 patients presented with functional symptoms. Of 6 patients who had functional symptoms, watery diarrhea was the most common symptom seen in $5(83.3 \%)$, followed by flushing (66.7\%), bronchospasm (16.7\%), and carcinoid heart disease $(16.7 \%)$. Among patients with GEP-NET, abdominal pain was the most common presenting symptom seen in $63.8 \%$ of cases, followed by vomiting (13.8\%), dyspepsia $(10.3 \%)$, jaundice $(10.3 \%)$, and weight loss $(6.9 \%)$. The overall spectrum of symptomatology in NETs is shown in Figure 2.

Overall, 66\% (64/97) of patients had de novo metastatic disease on presentation. Liver was the most common site of metastasis $(87.5 \%)$, followed by bone $(17.2 \%)$, lung $(6.25 \%)$, lymph nodes $(4.7 \%)$, adrenal glands $(1.6 \%)$, uterus $(1.6 \%)$, peritoneum $(1.6 \%)$, and brain $(1.6 \%)$. A strong correlation was noted between the primary site of origin and metastatic presentation $(P=0.016)$.

Of 97 patients, 31 (32\%) belonged to Grade 1 (low grade), $32(33 \%)$ belonged to Grade 2 (intermediate grade), and $34(35 \%)$ belonged to Grade 3 (high grade). One patient with ileal NET belonged to well-differentiated Grade 3 subset (MIB-1 index: 25\%). Among GEP-NETs, esophagus, large intestine, and rectum had predominantly high-grade disease (100\%, 66.7\%, and $60 \%$, respectively), whereas pancreas, stomach, and small intestine had predominantly low/intermediate-grade disease. Among non-GEP-NETs, urinary bladder, breast, and vaginal NETs were predominantly high grade (100\% each), whereas pulmonary, liver, gallbladder, ovarian, maxillary, and NETs with unknown primary were predominantly low/intermediate grade. Although metastatic disease at presentation was the most common with Grade 3 NETs $(76.5 \%)$ as compared to Grade $1(51.6 \%)$ and Grade $2(68.75 \%)$, on statistical analysis, we could not find a significant association between tumor grade and metastatic presentation $(P=0.99)$.

Treatment modalities and response assessment

Of 97 patients, 20 did not turn up after the initial diagnosis for definitive treatment planning and were declared lost to follow-up. Thus, the remaining 77 patients who received some form of planned treatment were included in the treatment response and survival analysis. Of these 77 patients, $31(40.2 \%)$ received systemic chemotherapy, 22 (28.6\%) underwent surgery, $12(15.6 \%)$ were planned for watchful waiting, $9(11.7 \%)$ received LAR somatostatin analogs, and $2(2.6 \%)$ received a combination of chemotherapy and LAR. One patient opted for peptide receptor radiotherapy (PRRT), after initial diagnosis and workup and received PRRT at a private

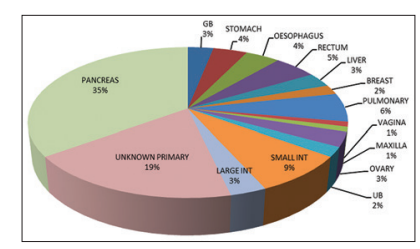

Figure 1: Pie chart showing primary site distribution of neuroendocrine tumors $(n=97)$

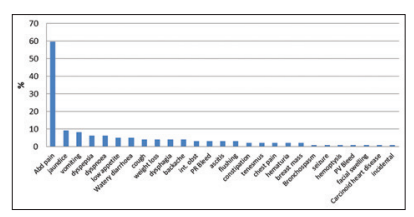

Figure 2: Spectrum of symptomatology of neuroendocrine tumors

institute and came for regular follow-up at our institute and so was included in the analysis [Table 1]. Of 31 patients who received chemotherapy, $25(80.64 \%)$ received a combination of carboplatin and etoposide and $5(16.1 \%)$ received a combination of capecitabine and temozolomide. Only one patient with primary breast NET received single-agent doxorubicin as first-line chemotherapy. Patients, who received systemic therapy as a primary treatment modality, received a median of six courses of carboplatin plus etoposide, three courses of capecitabine plus temozolomide (CAPTEM), seven courses of LAR, or six courses of chemotherapy plus LAR. Response assessment with individual treatment modalities is as follows.

\section{Carboplatin plus etoposide}

Among those patients who received carboplatin-etoposide combination, $3(12 \%)$ patients had complete response (CR), $12(48 \%)$ had partial response (PR), and $5(20 \%)$ had stable disease (SD). CR and PR were highest in Grade 3 NETs (18.8\% and 62.5\%, respectively) [Figures 3-5].

\section{Capecitabine plus temozolomide}

A total of 5 patients received CAPTEM combination as the first-line therapy ( 2 patients with Grade 2 NETs and 3 patients with Grade 3 NETs). Two (40\%) patients had PR and $1(20 \%)$ had SD, with no patient achieving CR. PR was seen more commonly in Grade 2 NETs $(50 \%)$ than Grade 3 NETs (33.3\%). Thus, CAPTEM combination chemotherapy showed the highest objective response rate (ORR) (50\%; 1 out of 2 Grade 2 NET patients) and the highest clinical benefit rate (CBR) in Grade 2 NET cases (100\%; 2 out of 2 cases) [Figures 3-5].

\section{Long-acting release Octreotide}

A total of 9 patients received LAR as an initial treatment with a median of six courses. Although there was no ORR, all patients had subjective improvement either in their functional or in their nonfunctional symptoms. Seven $(77.8 \%)$ patients had $\mathrm{SD}$ at a median follow-up of 8 months [Figures 3-5].

\section{Surgery}

A total of 22 patients underwent curative surgical resection. All patients who underwent surgery had complete (R0) resection with surgical CR rate of $100 \%$ irrespective of the grade of NETs. Three patients received adjuvant chemotherapy. At

Table 1: Treatment modalities offered to patients with neuroendocrine tumors $(n=77)$

\begin{tabular}{lc}
\hline Treatment modality & Frequency $(\%)$ \\
\hline Systemic CT & $31(40.2)$ \\
Surgery & $22(28.6)$ \\
Observation (watchful waiting) & $12(15.6)$ \\
Somatostatin analogs (LAR) & $9(11.7)$ \\
CT + LAR & $2(2.6)$ \\
PRRT & $1(1.3)$ \\
Total & $77(100)$ \\
\hline CT=Chemotherapy, LAR=Long-acting release, PRRT=Peptide receptor radiotherapy
\end{tabular}

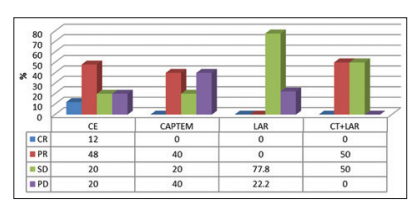

Figure 3: Composite bar diagram showing a response to various systemic treatments

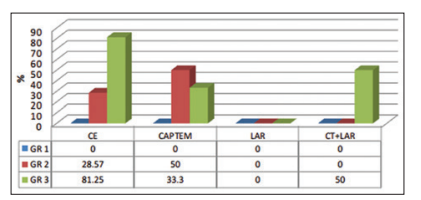

Figure 4: Composite bar diagram showing objective response rates of various systemic treatment regimens according to tumor grade 
a median follow-up of 10.75 months, 2 (9\%) patients had recurrent disease. Recurrence was seen only in Grade 3 NETs ( 2 out of 4 , i.e., $50 \%$ of Grade 3 NETs).

\section{Watchful waiting}

A total of 12 patients with Grade 1 and Grade 2 NETs were planned for close observation in view of minimal symptoms and were managed symptomatically. At a median follow-up of 8 months, 8 (66.7\%) of 12 patients had SD with good symptom control and quality of life on symptomatic medications.

\section{Survival analysis}

At the end of the median follow-up of 9 months (range: 3-24 months), only 20 (25.9\%) patients had documented radiological progressive disease at the time of the last follow-up. Hence, the median progression-free survival (PFS) was not reached. Treatment-wise subset analysis showed that EFS was highest, i.e., 10 months (range: 3-23 months) in the group undergoing curative surgery, whereas it was 7 months each for carboplatin and etoposide combination (range: 3-12 months) and octreotide LAR (range: 3-24 months), respectively. Close observation in the selected low/intermediate-grade NETs also showed considerably good EFS of 6.75 months (range: 3-18 months). Of 77 evaluable patients, 76 were alive at a median follow-up of 9 months (OS $=98.7 \%$ ) [Table 2].

\section{Discussion}

This study focuses on the epidemiology, clinicopathological characteristics, and therapeutic modalities applied in patients with all types of NETs, except small- and large-cell lung cancer. Various studies across the world have reported variations in the age, sex distribution, primary site, and patterns of disease presentation in Western countries and Asian regions. The median age of presentation of NETs reported in large Western studies is generally more than 60 years. ${ }^{[6,7]}$ Furthermore, the analyses of surveillance, epidemiology, and end results program (SEER) database and Norwegian studies have reported female preponderance. ${ }^{[6,7]}$ The median age of presentation in our study group was 49 years, which is younger as compared to Western literature. Furthermore, male predominance seen in our population can be because of reporting bias as males are more likely to present to health-care facilities in the Indian setup.

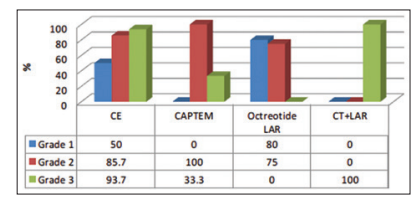

Figure 5: Composite bar diagram showing clinical benefit rates of various systemic treatment regimens according to tumor grade

Table 2: Event-free survival as per the primary treatment modality

\begin{tabular}{lccc} 
Treatment modality & $n$ & \multicolumn{2}{c}{ Months (range) } \\
\cline { 2 - 4 } & & Median follow-up & Median EFS \\
\hline Carboplatin + etoposide & 25 & $9.5(3-18)$ & $7(3-12)$ \\
CAPTEM & 5 & $7.5(3-12)$ & $3(3-8)$ \\
Doxorubicin & 1 & 10 & 9.5 \\
Octreotide LAR & 9 & $8(3-24)$ & $7(3-24)$ \\
Chemotherapy + LAR & 2 & $10.5(9-12)$ & $8.5(8-9)$ \\
Surgery & 22 & $10.75(3-24)$ & $10(2-23)$ \\
Observation & 12 & $8(3-24)$ & $6.75(3-18)$ \\
PRRT & 1 & 4 & 3 \\
\hline LAR=Long-acting release, PRRT=Peptide receptor radiotherapy, EFS=Event-free \\
survival, CAPTEM=Capecitabine plus temozolomide \\
$\mathbf{2 0 0}$
\end{tabular}

The largest SEER analysis reports lung as the most common site of primary NET, whereas Norwegian study ${ }^{[7]}$ and Taiwanese study ${ }^{[8]}$ found small intestine and rectum as the most common primary sites, respectively. In an Indian retrospective analysis of GEP-NETs, the most common site of primary tumor was stomach $(30.2 \%)$, followed by pancreas $(23.3 \%){ }^{[9]}$ However, in an Iraqi study ${ }^{[10]}$ and an another recent Indian study by Kapoor et al. ${ }^{[11]}$ pancreas was the most common primary site seen in $26.3 \%$ and $35.2 \%$ cases, respectively, which is similar to our study [Table 3]. Overall, the above studies showed that there are possible ethnic and regional variations in the primary site of origin of NETs, with pancreatic NETs being more common as compared to Western literature.

Overall, functional NETs were less common in our study group, which is not typically seen in a Western population where the reported incidence of functional NETs ranged from $20 \%$ to $30 \% \cdot{ }^{[12]}$ However, previous Indian study of GEP-NETs found only $4.1 \%$ of patients presenting with functional symptoms similar to our study. ${ }^{[1]]}$ This may represent some differences in tumor biology in the Indian population.

The main issue in the Indian population is delayed presentation and diagnosis at advanced stages. This is contrary to the largest Western data of SEER analysis where only $21 \%$ of cases had distant metastasis on presentation. ${ }^{[6]}$ Similarly, various other studies have reported de novo metastatic rates ranging from $20.8 \%$ to $53.1 \%$, which is less as compared to our study. ${ }^{[10,12,13]}$ A higher proportion of metastatic disease at presentation may be probably because of delayed presentation to the health-care facilities in our country and nonspecific nature of symptoms leading to delayed diagnosis.

In our study, the nature of initial presentation (metastatic vs. nonmetastatic) varied according to the site of origin of NET. There was a strong correlation between the primary site of origin and metastatic presentation $(P=0.016)$. Metastatic disease at presentation was more common in NETs of large intestine $(100 \%)$, gallbladder $(100 \%)$, breast $(100 \%)$, rectum $(80 \%)$, and stomach $(75 \%)$ [Table 4$]$. This may be because of early symptomatic presentation in these cases, leading to early diagnosis as compared to GEP-NETs where the diagnosis is often delayed due to nonspecific symptoms. A similar association between the primary site of origin of NET and the stage at diagnosis has been reported in the largest SEER analysis and analysis from the Netherlands Cancer Registry.

Recent studies have shown that a small subset of patients differentiated are associated with high Ki67 proliferation indices $(>20 \%)$ thus falling into the high grade (G3) range in the current WHO grading scheme. The clinical behavior of these grade-discordant tumors is somewhat worse than grade-concordant well-differentiated G2 tumors, but better than that of bona fide poorly differentiated NECs. In limited literature available, the incidence of well-differentiated Grade 3 NETs has been reported between $5.6 \%$ and $8 \%$ of the GEP-NETs in various studies. ${ }^{[14,15]}$ In our study group, we had only one case of ileal NET ( $1 \%$ overall and $1.7 \%$ of GEP-NETs) with histologically low-grade NET with low mitosis, but had Ki67 index 25\%, thus fitting into the category of well-differentiated high-grade NET.

Response to chemotherapy also varies according to chemotherapy regimens and histological characteristics of South Asian Journal of Cancer Volume 8 Issue 3 July-September 2019 
Table 3: Top 5 primary sites of origin in various studies and comparison with our study

\begin{tabular}{|c|c|c|c|c|c|c|c|}
\hline $\begin{array}{l}\text { Primary } \\
\text { site rank }\end{array}$ & Our study & $\begin{array}{l}\text { Yao JC et al. }{ }^{[10]} \\
\text { (US Whites) }\end{array}$ & $\begin{array}{l}\text { Yao JC et al. }{ }^{[10]} \\
\text { (US Asians/PI) }\end{array}$ & $\begin{array}{l}\text { Hauso O } \\
\text { et al. }{ }^{[11]}\end{array}$ & Tsai HJ et al..$^{[12]}$ & $\begin{array}{l}\text { Abdulfattah MK } \\
\text { et al. }{ }^{[14]}\end{array}$ & Kapoor $\mathrm{R}$ et al..$^{[15]}$ \\
\hline 1 & Pancreas $(35 \%)$ & Lung $(30 \%-32 \%)$ & Rectum $(41 \%)$ & $\begin{array}{l}\text { Small intestine } \\
(26 \%)\end{array}$ & Rectum $(25 \%)$ & Pancreas $(26.3 \%)$ & Pancreas $(35.2 \%)$ \\
\hline 2 & $\begin{array}{l}\text { Unknown } \\
\text { primary }(19 \%)\end{array}$ & $\begin{array}{l}\text { Small intestine } \\
(18 \%-19 \%)\end{array}$ & Lung $(15 \%)$ & Lung $(21 \%)$ & Lung $(20 \%)$ & Pelvis $(15.8 \%)$ & Periampullary $(21.5 \%)$ \\
\hline 3 & $\begin{array}{l}\text { Small intestine } \\
(9 \%)\end{array}$ & $\begin{array}{l}\text { Unknown primary } \\
(13 \%)\end{array}$ & Pancreas $(8 \%)$ & Colon $(8 \%)$ & Stomach $(7 \%)$ & Lung (13.2\%) & Small intestine $(13.7 \%)$ \\
\hline 4 & Lung $(6 \%)$ & Rectum $(12 \%)$ & $\begin{array}{l}\text { Small intestine } \\
(8 \%)\end{array}$ & Rectum $(7 \%)$ & Pancreas $(6 \%)$ & $\begin{array}{l}\text { Small intestine } \\
(13.2 \%)\end{array}$ & Retroperitoneum (9.8\%) \\
\hline 5 & Rectum $(5 \%)$ & Colon $(7 \%-8 \%)$ & Stomach $(6 \%)$ & Pancreas $(7 \%)$ & Colon $(5 \%)$ & $\begin{array}{l}\text { Mediastinum } \\
(10.5 \%)\end{array}$ & $\begin{array}{l}\text { Unknown primary } \\
(9.8 \%)\end{array}$ \\
\hline
\end{tabular}

Table 4: Primary site and metastatic potential

\begin{tabular}{lccc}
\hline Primary site & $n$ & Metastatic (\%) & Nonmetastatic (\%) \\
\hline Pancreatic & 34 & $18(53)$ & $16(47)$ \\
Unknown primary & 18 & $18(100)$ & $0(0)$ \\
Small intestine & 8 & $6(75)$ & $2(25)$ \\
Pulmonary & 6 & $3(50)$ & $3(50)$ \\
Rectum & 5 & $4(80)$ & $1(20)$ \\
Stomach & 4 & $3(75)$ & $1(25)$ \\
Esophagus & 4 & $1(25)$ & $3(75)$ \\
Large intestine & 3 & $3(100)$ & $0(0)$ \\
Liver & 3 & $1(33.3)$ & $2(66.7)$ \\
Gallbladder & 3 & $3(100)$ & $0(0)$ \\
Ovary & 3 & $2(66.7)$ & $1(33.3)$ \\
Urinary bladder & 2 & $0(0)$ & $2(100)$ \\
Breast & 2 & $2(100)$ & $0(0)$ \\
Vagina & 1 & $0(0)$ & $1(100)$ \\
Maxilla & 1 & $0(0)$ & $1(100)$ \\
Total & 97 & 64 & 33 \\
\hline
\end{tabular}

NETs. The objective response rates for platinum and etoposide combination have been reported ranging from $47.4 \%$ to $68 \%$ and that too maximum in poorly differentiated NETs than well-differentiated NETs. ${ }^{[16-18]}$ On similar lines, our study suggests that carboplatin and etoposide is more effective in high-grade NETs than low-grade NETs.

With CAPTEM regimen, PR was seen more commonly in Grade 2 NETs (50\%) than Grade 3 NETs (33.3\%). In a similar study by Ramirez et al., ${ }^{[18]}$ CBR rates were $76 \%$, 69\%, and $40 \%$ in Grade 1, Grade 2, and Grade 3 NETs, respectively. The highest response rate for CAPTEM regimen has been reported as $70 \%$ in pancreatic NETs, which is higher than that seen in our study. ${ }^{[19]}$ Overall, our study suggests that CAPTEM is more effective in low/intermediate-grade NETs than high-grade NETs.

With somatostatin analogs/LAR, though there was no ORR, all patients had subjective symptomatic improvement. Seven (77.8\%) patients had SD at a median follow-up of 8 months, which is similar to that observed in PROMID study. ${ }^{[20]}$

There are certain limitations in our study. The most important limitation was a shorter follow-up, and many patients were lost to follow-up after subsequent response evaluation because of which median PFS could not be reached. These patients were included in EFS analysis with the event truncated at the last follow-up. Furthermore, DOTANOC scanning, serum chromogranin A testing, and newer treatment modalities such as PRRT were not available due to limited resources. Larger multicentric data with a longer follow-up are essentially required for better defining of disease characteristics in the Indian population.

South Asian Journal of Cancer $\bullet$ Volume 8 • Issue 3 • July-September 2019

\section{Conclusions}

The clinical profile of NETs in the Indian population is different as compared to Western countries. Although previous Indian study has shown stomach as the most common primary site, recent Indian studies have shown pancreas as the most common primary site of origin, suggesting that the epidemiological profile of NETs in India is changing or a possible regional variation. Functional NETs are less common, suggesting a different biological profile in the Indian population. Carboplatin-etoposide combination chemotherapy remains most effective in high-grade metastatic NECs, whereas CAPTEM is most effective in Grade 2 NETs. Thus, CAPTEM can be a cost-effective alternative to octreotide LAR in patients with nonfunctional low/intermediate-grade well-differentiated NETs. PRRT remains an expensive and less accessible option for patients in our setup. There is an urgent unmet need for creating awareness regarding the symptomatology, diagnostic modalities, and formulating Indian NET guidelines for optimal treatment of our patients. More multi-institutional studies are required to better define the epidemiological and clinical profile of this "rare" but "not so rare" disease.

\section{Financial support and sponsorship}

Nil.

\section{Conflicts of interest}

There are no conflicts of interest.

\section{References}

1. Norton JA, Kunj PL. Carcinoid tumors and the carcinoid syndrome. In: DeVita VT Jr., Hellman S, Rosenberg SA, editors. Cancer: Principles \& Practice of Oncology. $10^{\text {th }}$ ed. Philadelphia, PA: Lippincott Williams \& Wilkins; 2015. p. 1218-26.

2. Taal BG, Visser O. Epidemiology of neuroendocrine tumours. Neuroendocrinology 2004;80 Suppl 1:3-7.

3. Rindi G, Klöppel G, Alhman H, Caplin M, Couvelard A, de Herder WW, et al. TNM staging of foregut (neuro) endocrine tumors: A consensus proposal including a grading system. Virchows Arch 2006;449:395-401.

4. Rindi G, Klöppel G, Couvelard A, Komminoth P, Körner M, Lopes JM, et al. TNM staging of midgut and hindgut (neuro) endocrine tumors: A consensus proposal including a grading system. Virchows Arch 2007;451:757-62.

5. Rindi G, Petrone G, Inzani F. The 2010 WHO classification of digestive neuroendocrine neoplasms: A critical appraisal four years after its introduction. Endocr Pathol 2014;25: 186-92.

6. Yao JC, Hassan M, Phan A, Dagohoy C, Leary C, Mares JE, et al. One hundred years after "carcinoid": Epidemiology of and prognostic factors for neuroendocrine tumors in 35,825 cases in the United States. J Clin Oncol 2008;26:3063-72.

7. Hauso O, Gustafsson BI, Kidd M, Waldum HL, Drozdov I, Chan AK, et al. Neuroendocrine tumor epidemiology: Contrasting Norway and North America. Cancer 2008; 113:2655-64.

8. Tsai HJ, Wu CC, Tsai CR, Lin SF, Chen LT, Chang JS. The epidemiology of neuroendocrine tumors in Taiwan: A nation-wide cancer registry-based study. PLoS One 2013;8:e62487.

9. Amarapurkar DN, Juneja MP, Patel ND, Amarapurkar AD, Amarapurkar PD. 
A retrospective clinico-pathological analysis of neuroendocrine tumors of the gastrointestinal tract. Trop Gastroenterol 2010;31:101-4.

10. Abdulfattah MK, Al-Naqqash MA. The clinico-epidemiologic characteristics of Iraqi patients with neuroendocrine tumors and their response to long acting octreotide. J Fac Med Baghdad 2016;58:312-5.

11. Kapoor R, Bhattacharyya T, Gupta R, Mitta BR, Kalra N. A systematic review of management of neuroendocrine tumors: An experience from a tertiary care centre from India. Clin Cancer Investig J 2014;3:363-72.

12. Hafeez U, Joshi A, Bhatt M, Kelly J, Sabesan S, Vangaveti V. Clinical profile and treatment outcomes of advanced neuroendocrine tumours in rural and regional patients: A retrospective study from a regional cancer centre in North Queensland, Australia. Intern Med J 2017;47:284-90.

13. Hallet J, Law CH, Cukier M, Saskin R, Liu N, Singh S, et al. Exploring the rising incidence of neuroendocrine tumors: A population-based analysis of epidemiology, metastatic presentation, and outcomes. Cancer 2015; 121:589-97.

14. Vélayoudom-Céphise FL, Duvillard P, Foucan L, Hadoux J, Chougnet CN, Leboulleux S, et al. Are G3 ENETS neuroendocrine neoplasms heterogeneous? Endocr Relat Cancer 2013;20:649-57.

15. Basturk O, Yang Z, Tang LH, Hruban RH, Adsay V, McCall CM, et al. The high-grade (WHO G3) pancreatic neuroendocrine tumor category is morphologically and biologically heterogenous and includes both well differentiated and poorly differentiated neoplasms. Am J Surg Pathol 2015;39:683-90.

16. Imai H, Shirota H, Okita A, Komine K, Saijo K, Takahashi M, et al. Efficacy and safety of carboplatin and etoposide combination chemotherapy for extrapulmonary neuroendocrine carcinoma: A retrospective case series. Chemotherapy 2016;61:111-6.

17. Bongiovanni A, Riva N, Ricci M, Liverani C, La Manna F, De Vita A, et al. First-line chemotherapy in patients with metastatic gastroenteropancreatic neuroendocrine carcinoma. Onco Targets Ther 2015;8:3613-9.

18. Ramirez RA, Beyer DT, Chauhan A, Boudreaux JP, Wang YZ, Woltering EA. The role of capecitabine/temozolomide in metastatic neuroendocrine tumors. Oncologist 2016;21:671-5.

19. Strosberg JR, Fine RL, Choi J, Nasir A, Coppola D, Chen DT, et al. First-line chemotherapy with capecitabine and temozolomide in patients with metastatic pancreatic endocrine carcinomas. Cancer 2011;117:268-75.

20. Rinke A, Müller HH, Schade-Brittinger C, Klose KJ, Barth P, Wied M, et al. Placebo-controlled, double-blind, prospective, randomized study on the effect of octreotide LAR in the control of tumor growth in patients with metastatic neuroendocrine midgut tumors: A report from the PROMID study group. J Clin Oncol 2009;27:4656-63. 